\title{
Improving the Navigation System of a UAV Using Multi-Sensor Data Fusion Based on Fuzzy C-Means Clustering
}

\author{
A. D. Abosekeen ${ }^{*}$, A. E. Abdalla ${ }^{\dagger}$
}

\begin{abstract}
In this paper a multi-sensor data fusion technique is applied to aerosonde unmanned aerial vehicle (UAV) model to enhance inertial navigation system. An inertial measuring unit (IMU) error model is built with different error parameters (biasing, scale factor, and noise). Each IMU output is applied to strap down inertial navigation system (INS) algorithm to obtain the navigation information: position, velocity, and attitude (PVA). Multisensor data fusion algorithm based on fuzzy c-means clustering (FCM) is used to fuse the IMUs data. The fused output is applied to the INS algorithm to obtain the PVA. The simulation results show the effectiveness of the proposed method in reducing the error in navigation information PVA than using a single IMU.
\end{abstract}

Keywords: Data fusion, fuzzy, data clustering, inertial navigation

\section{Introduction}

Over the past two decades, significant attention has been focused on multi-sensor data fusion for both military and civilian applications. Data fusion techniques combine data from multiple sensors and related information to achieve more specific inferences than could be achieved by using a single, independent sensor[1]. Fusion processes are often categorized in three levels of modes (low, intermediate, and high level fusion), as follows[2]:

1. Low level fusion combines several sources of essentially the same type of raw preprocessed data to produce a new data set that is expected to be more informative and useful than the inputs.

2. Intermediate level, mid-level fusion, or feature level fusion combines various features such as edges, lines, corners, textures, or positions into a feature map. This map is used for segmentation of images, detection of objects, etc. This process of fusion is called pixel, feature, or image level fusion.

3. High level fusion, or decision fusion, combines decisions from several experts. Methods of decision fusion are voting, fuzzy logic, and statistical methods. The present study is interested in some aspects of this level of fusion, mainly fuzzy logic based decision fusion.

The conventional approaches for multi-sensor data fusion like weighted average, Bayesian estimators, adaptive observers, algebraic functions, fuzzy logic, neural network, soft computing, nonlinear system fusion, and Kalman filtering are suffering from a lot of problems such as:

\footnotetext{
* Egyptian Armed Forces, Egypt. ashraf.sekeen@gmail.com

${ }^{\dagger}$ Egyptian Armed Forces, Egypt.
} 
1. The necessity of adding new sensors to the system.

2. Use linear estimation models that need a previous knowledge of signal statistics.

3. The presence of more than one faulty signal is an essential limitation of the performance.

4. Requirement of knowledge of the behavior of the system to generate the governing rules.

The paper objective is producing a multi-sensor data fusion method that can overcome most of these problems. This method consists of two stages as follows:

1. Separating the sensors data by using Fuzzy C-Means (FCM) algorithm as a data clustering method.

2. A single output based on the information that comes from the sensors is produced by the fusion algorithm.

This method will be detailed in section (4). Using multi-sensor data fusion in aircraft navigation applications has appeared in recent years with the advent of low cost, small size and low mass navigation sensors (e.g. optical gyros, MEMS inertial sensors and GNSS sensors), [3].

Inertial navigation system model based on quaternions [4-8] is used for the navigation process of the Aerosonde UAV model, [9]. The Inertial sensors are 3 IMUs used for measure the rates and accelerations of the UAV, an error model is added to the true raw data from the UAV such that the measurements are as MEMS sensors, and by changing the error parameters in the error model three coincident IMUs are simulated to be used in navigation process. This error model is explained in the next section.

\section{IMU Error Model}

For simulating IMU measurement, an error model shown in Figure 1 is applied [10], where: $N=\left(0, \sigma^{2}\right)$ denotes a zero-mean normal distribution with $\sigma^{2}$, the error model consists of: (gyroscope error model + accelerometer error model).

\section{The Gyro Error Model}

$\tilde{\omega}_{i b}^{b}=\left(I_{3 \times 3}+s_{g}\right) \omega_{i b}^{b}+b_{g}+\eta_{g v}$

$\dot{b}_{g}=\eta_{g u}$

where: $b_{g}$ is the gyro bias, $s_{g}$ is a diagonal matrix of gyro scale factors, $\eta_{\mathrm{gv}}$ and $\eta_{g u}$ are zero-mean Gaussian white-noise processes with spectral densities given by $\sigma_{g v}^{2} I_{3 \times 3}$ and $\sigma_{g u}^{2} I_{3 \times 3}$, respectively.

\section{The Accelerometer Error Model}

The same model can be applied to the accelerometers as

$\tilde{a}^{b}=\left(I_{3 \times 3}+s_{a}\right) a^{b}+b_{a}+\eta_{a v}$

$\dot{b}_{a}=\eta_{a u}$

where: $b_{a}$ is the accelerometer bias, $s_{a}$ is a diagonal matrix of accelerometer scale factors $\eta_{a v}$, and $\eta_{a u}$ are zero-mean Gaussian white-noise processes with spectral densities given by $\sigma_{a v}^{2} I_{3 \times 3}$ and $\sigma_{a u}^{2} I_{3 \times 3}$ respectively. 


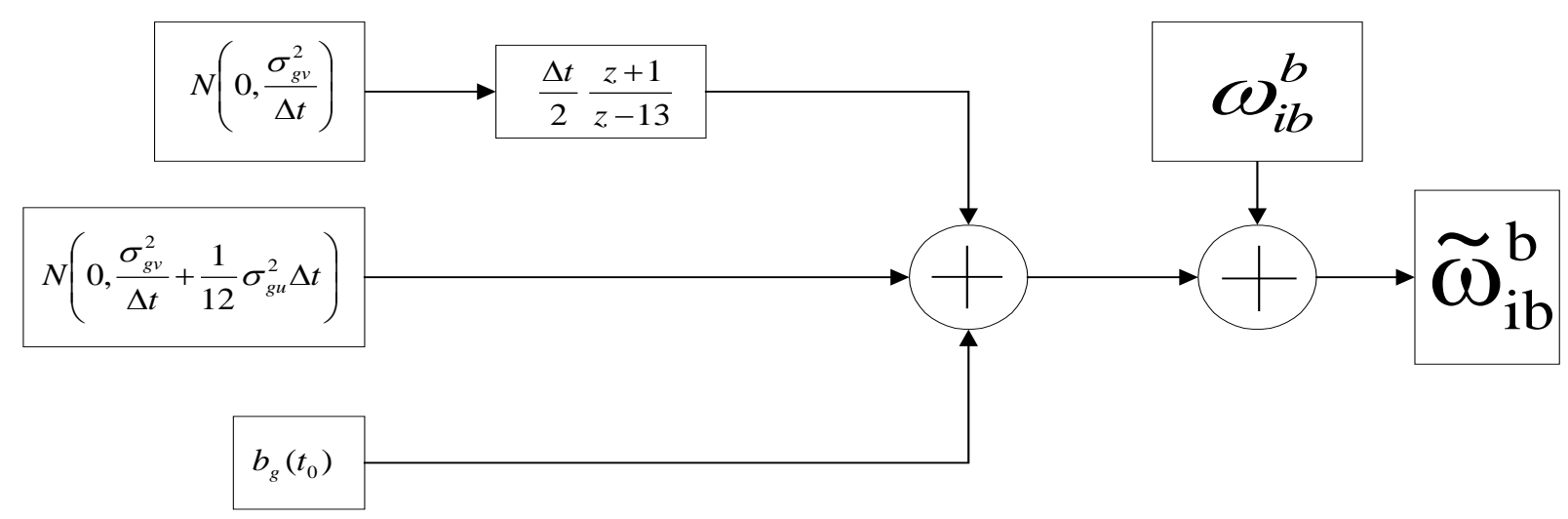

Figure 1. Gyroscope Error Model

\section{Aerosonde UAV Navigation}

The block diagram shown in Figure 2 shows that the aircraft model outputs are the true (position, velocity, and attitude ) PVA and the IMU measurements (accelerations, gyroscopes rates) the true PVA is the reference data that after applying the IMU measurements to the INS algorithm the output PVA will be compared with it, also three error models are added to the IMU measurements from the aircraft model simulating 3 different IMUs, each of them is applied to the INS algorithm and the outputs are compared with the true PVA from the aircraft model.

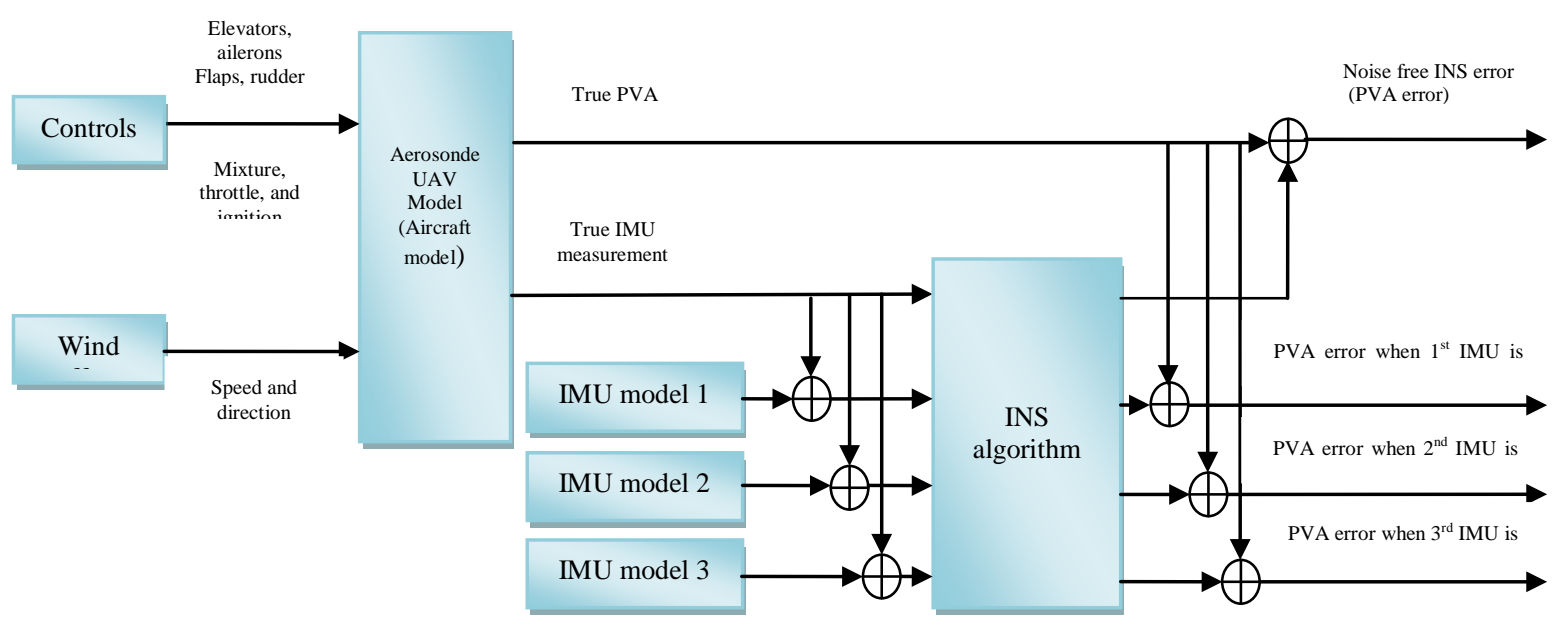

Figure 2. The Navigation Using Error Free and Error Models Addition to 3 IMUs

\section{Multi-Sensor Data Fusion}

The process of combining the provided information from multiple sensors is called sensor fusion, and can overcome a number of problems ranging from noise to incipient sensor failure. Even in the absence of these issues, one can increase the system's accuracy and the reliability using sensor fusion[11]. The U.S. Department of Defense conducted much of the early research on this technology and explored its usefulness in military surveillance and land-based battle management systems. Many papers and books talk about various techniques and algorithms for data fusion such as least square method, Bayesian method, Kalman filter method, fuzzy logic, and neural network[2] . This paper presents architecture for fusing data obtained from several IMUs based on FCM. 


\subsection{Fuzzy C-Means Clustering Algorithm (FCM)}

Cluster analysis refers to a lot of methods which aim to subdivide a data set $\mathrm{X}$ into $\mathrm{C}$ subsets (clusters). Fuzzy clustering plays an important role in solving problems in the areas of pattern classification, fuzzy intelligent control and to classify fault patterns. Fuzzy c-means (FCM) proposed by Bezdek (1981) is one of the most famous techniques in clustering analysis [1216]. FCM clustering is depending on the measure of distance between samples. Generally, FCM uses the common Euclidean distance. The FCM allows each feature vector to belong to every cluster with a fuzzy truth value (between 0 and 1 ). The objective of FCM is to obtain the fuzzy c-partition $\tilde{F}=\left\{\underset{1}{\tilde{F}}, \tilde{F}_{2}, \ldots ., \tilde{F}_{c}\right\}$ for both data set $X=\left\{x_{1}, \ldots \ldots ., x_{n}\right\}$ and the number of clusters c by minimizing $J_{m}$

Minimize $J_{m}(U, V: X)=\sum_{i=1}^{c} \sum_{j=1}^{n}\left(\mu_{i j}\right)^{m}\left\|X_{j}-v_{i}\right\|^{2}$

where $\mu_{i j}$ the membership degree of data is point $x_{j}$ to the fuzzy cluster $\underset{i}{\tilde{F}}$, and is also an element of a $(c \times n)$ pattern matrix $U=\left[\mu_{i j}\right]$. The $i^{t h}$ row of $U, U_{i}$, corresponding to a fuzzy cluster $\underset{i}{\tilde{F}} \cdot V=\left(v_{1}, v_{2}, \ldots ., v_{c}\right)$ is a vector of cluster centroids of the fuzzy cluster $\{\underset{1}{\tilde{F}}, \underset{2}{\tilde{F}}, \ldots ., \underset{c}{\tilde{F}}\}$. Thus, a fuzzy partition can be denoted by the pair $(U, V)$. $\left\|x_{j}-v_{i}\right\|$ is the Euclidean norm between $x_{j}$ and $v_{i}$. The parameter m controls the fuzziness of membership of each datum. The complete fusion mechanization will be as Figure 3:

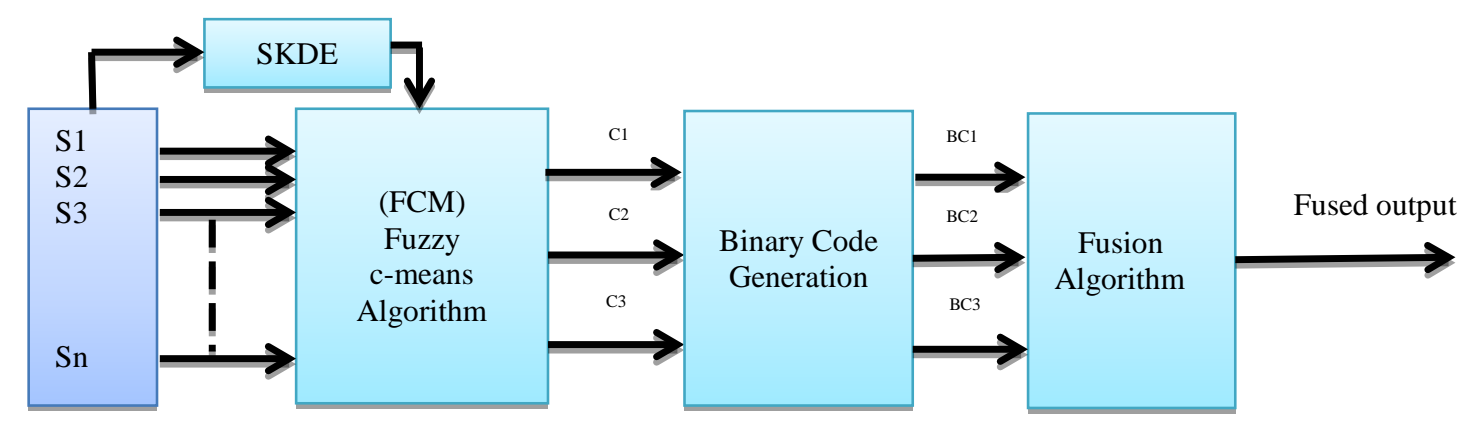

Figure 3 Complete Fusion Mechanization

\subsection{Steps of Data Clustering Using FCM Algorithm}

1. As shown in Figure 4 a pre-selected number of clusters $C$, and a chosen value of $\mathrm{m}$, initialize the membership matrix $U=\left[\mu_{i j}\right]$ of $x_{j}$ belonging to cluster $\underset{i}{\tilde{F}}$ (for $i=1,2, \ldots, c$ with random numbers whose values are between 0 and 1 and make it satisfy the constraint condition below,

$$
\sum_{i=1}^{c} \mu_{i j}=1
$$

2. Compute the fuzzy cluster centroid $v_{i}$ for $i=1,2, \ldots, c$ using 
$v_{i}=\frac{\sum_{j=1}^{n}\left(\mu_{i j}\right)^{m} x_{j}}{\sum_{j=1}^{n}\left(\mu_{i j}\right)^{m}}$

3. Calculate the value of the function $J_{m}(U, V)$. If it is less than a certain threshold $(\varepsilon)$ value $\left\{\left|v^{t}-v^{t-1}\right|\right\}<\varepsilon$ or compared with the last value function, its variation of function value is less than a certain threshold value, this algorithm stops.

4. Update the fuzzy membership $U=\left[\mu_{i j}\right]$ for $i=1,2, \ldots, c$ using the following formula, Then return to step 2 .

$\mu_{i j}=\left[\sum_{k=1}^{c}\left(\frac{\left\|X_{j}-v_{i}\right\|^{2}}{\left\|x_{j}-v_{k}\right\|^{2}}\right)^{1 / m-1}\right]^{-1}$

According to the above discussion, FCM algorithm requires predefining the number of clusters (c) as in Figure 3. In this paper the number of clusters is computed based on the function proposed by the smooth kernel density estimator (SKDE) $[17,18]$ which is obtained as follows:

$p(s)=\frac{1}{m h} \sum_{i=1}^{m} k\left(\frac{s-s_{i}}{h}\right)$

$k(s)=\frac{1}{(2 \pi)^{1 / 2}} e^{\left(-\frac{1}{2} s^{T} s\right)}$

where $h$ is the length of the estimation window. The number of the clusters is determined according to the following two rules:

1. If the maximum peak of the kernel estimator is left or right skewed then $\mathrm{C}=2$.

2. If the maximum peak of the kernel estimator is centered then $\mathrm{C}=3$.

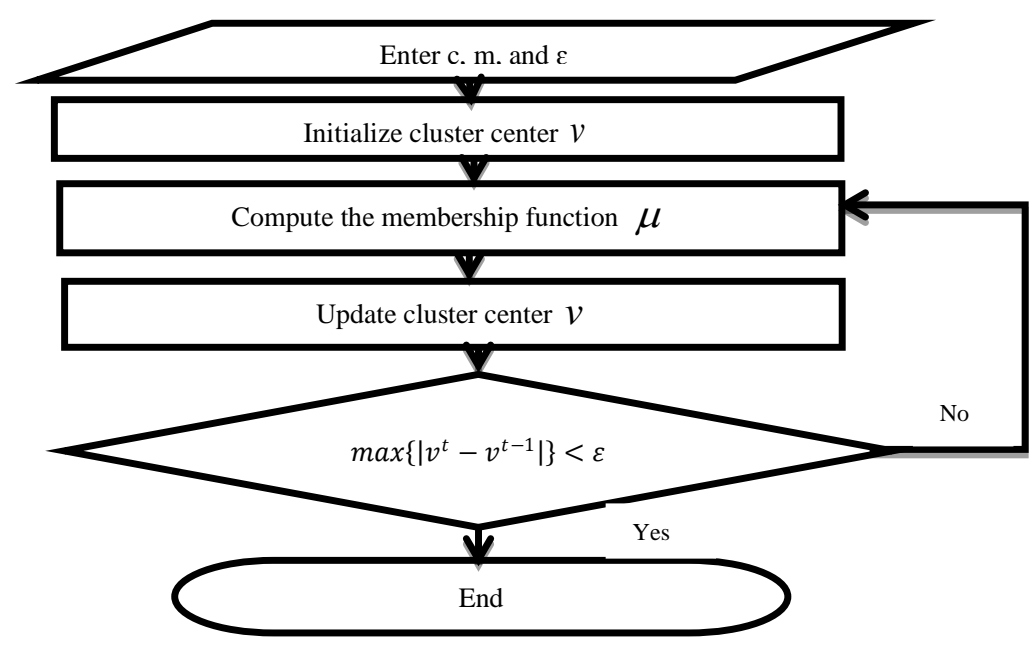

Figure 4. FCM Flowchart 


\subsection{Fusion Algorithm}

After the clustering process as shown in Figure 3 each cluster membership function is represented as a binary code $B_{C i} \in 2^{m}$. The creation of this code depends upon the membership functions for the clusters and a variable threshold level such that[16]:

$B_{C i}(S)= \begin{cases}1, & \text { if } \mu(s)<\alpha \\ 0, & \text { if } \mu(s)>\alpha\end{cases}$

where $(\alpha)$ is given as follows:

$\alpha=1-\frac{1}{m} \sum_{j=1}^{c} \sum_{i=1}^{m} \mu_{j}\left(s_{i}\right) \log \mu_{j}\left(s_{i}\right)$

The fused output will be the cluster center $v$ with the minimum binary code $B_{C i}$ that achieves the following minimization argument:

$s_{f}=v\left(\mu_{i^{*}}\right) \quad, i^{*}=\operatorname{argmin}\left(B_{c 1}, B_{c 2}, \ldots \ldots, B_{c i}\right)$

\section{Performance Evaluation and Simulation Results}

The proposed approach will be obtained by applying the multi-sensor data fusion algorithm to the output of three IMUs and comparing the PVA by using each IMU and by using the fused data for navigation. The complete block diagram will be as shown in Figure 5:

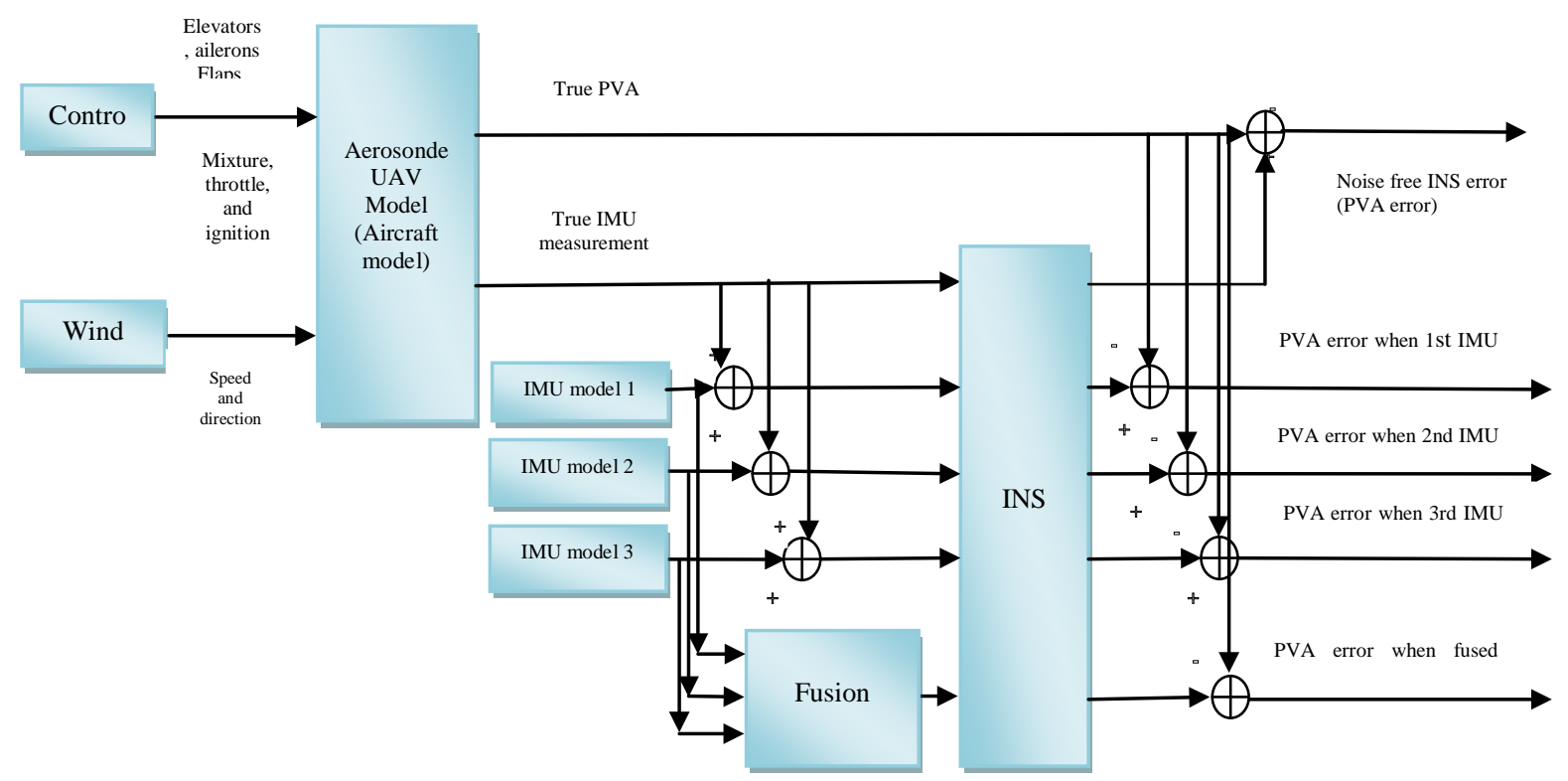

Figure 5. Complete block diagram of the fusion and navigation systems

A comparison of using single IMU navigation system over a certain path and the use of multiple IMU navigation system is shown in the following error figures (Position, Velocity, Attitude) errors respectively and the $2 \mathrm{D}$ path comparison is also shown as follows: 


\section{The Position Errors Figures}

1. The latitude error

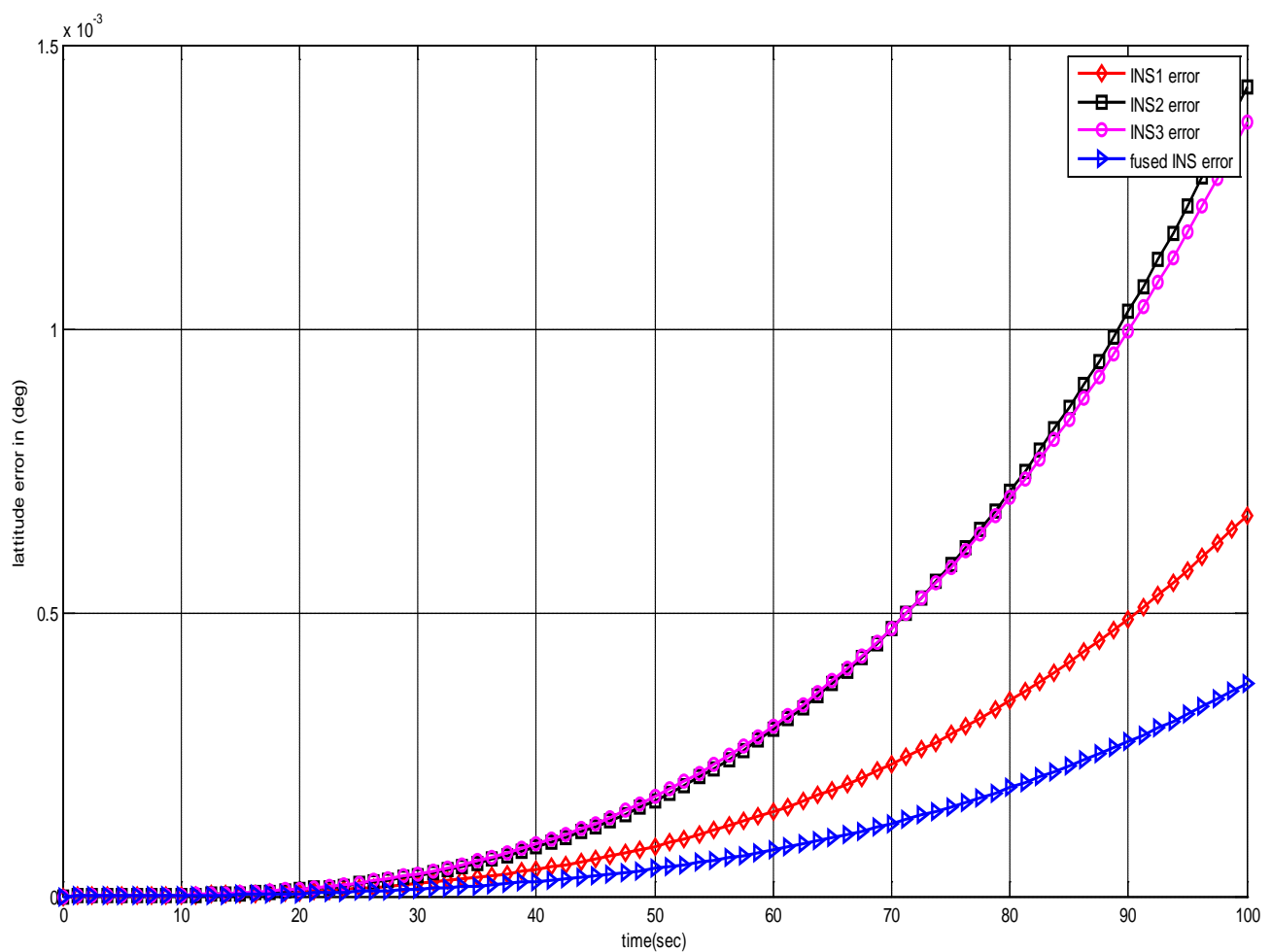

Figure 6. The latitude error

2. The longitude error

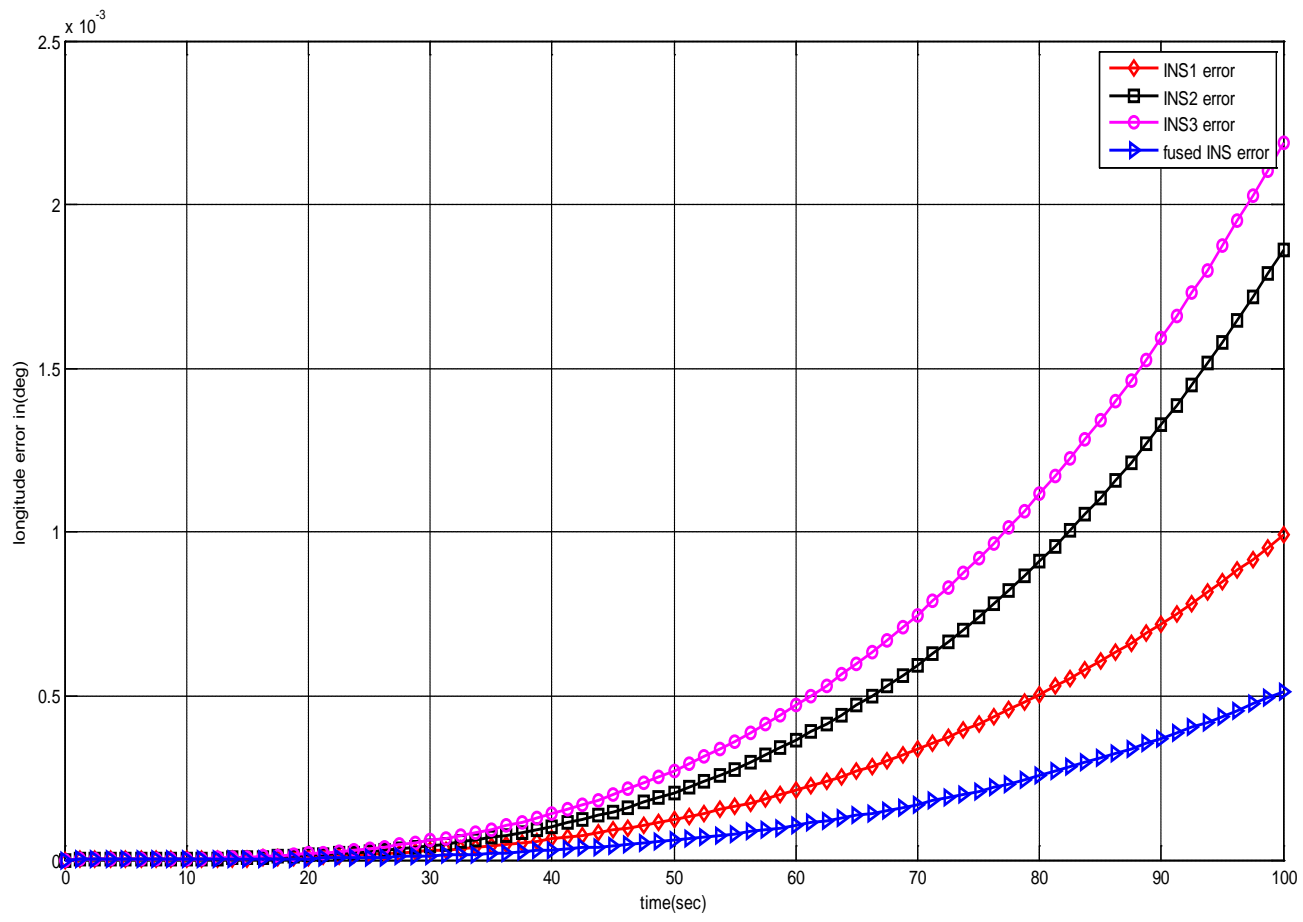

Figure 7. The longitude error 


\section{The altitude error}

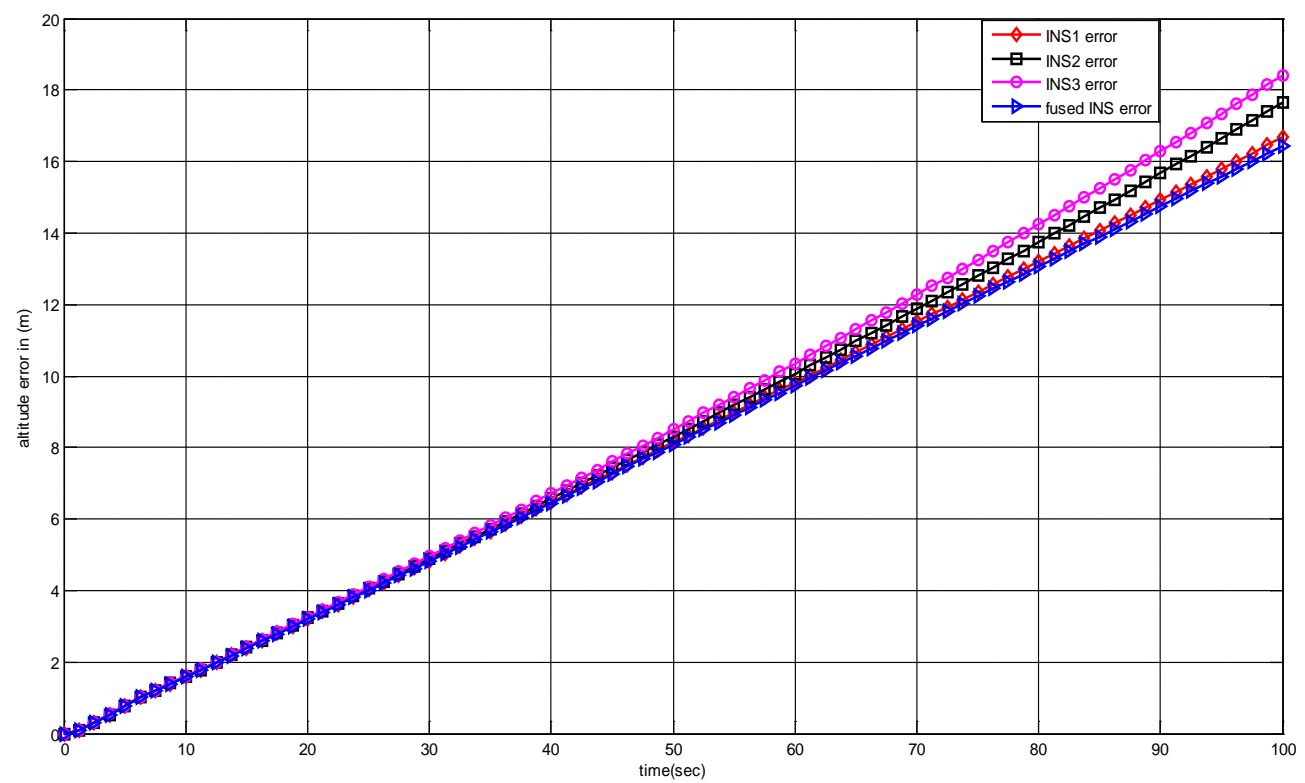

Figure 8. The altitude error

As shown in Figures (6, 7, and 8) the position errors (latitude, longitude, altitude) are increasing with time because the error of the INS is accumulated, but the error due to the use of the $1^{\text {st }}$ IMU , $2^{\text {nd }}$ IMU , and $3^{\text {rd }}$ IMU is increasing in a higher rate than the error of using the fusion of them. These results indicate that the use of multi-sensor data fusion in the navigation is better than the use of a single sensor.

Figure 9 shows that over the simulation period the error in the $\mathrm{x}$ direction using the fusion is about $24 \mathrm{~m}$ after $100 \mathrm{sec}$ and the errors using the IMUs 1, 2, and 3 are 45, 73, 98m respectively; the error in y direction using the fusion is about $37 \mathrm{~m}$; the errors using the IMUs 1,2 , and 3 are $77,164,171 \mathrm{~m}$ respectively; the error in $\mathrm{z}$ direction using the fusion is about $41 \mathrm{~m}$; and the errors using the IMUs 1, 2, and 3 are $64,120,124 \mathrm{~m}$ respectively. These results indicate that using fusion between the 3 IMUs produces the minimum error in the position and improves the performance of the INS.
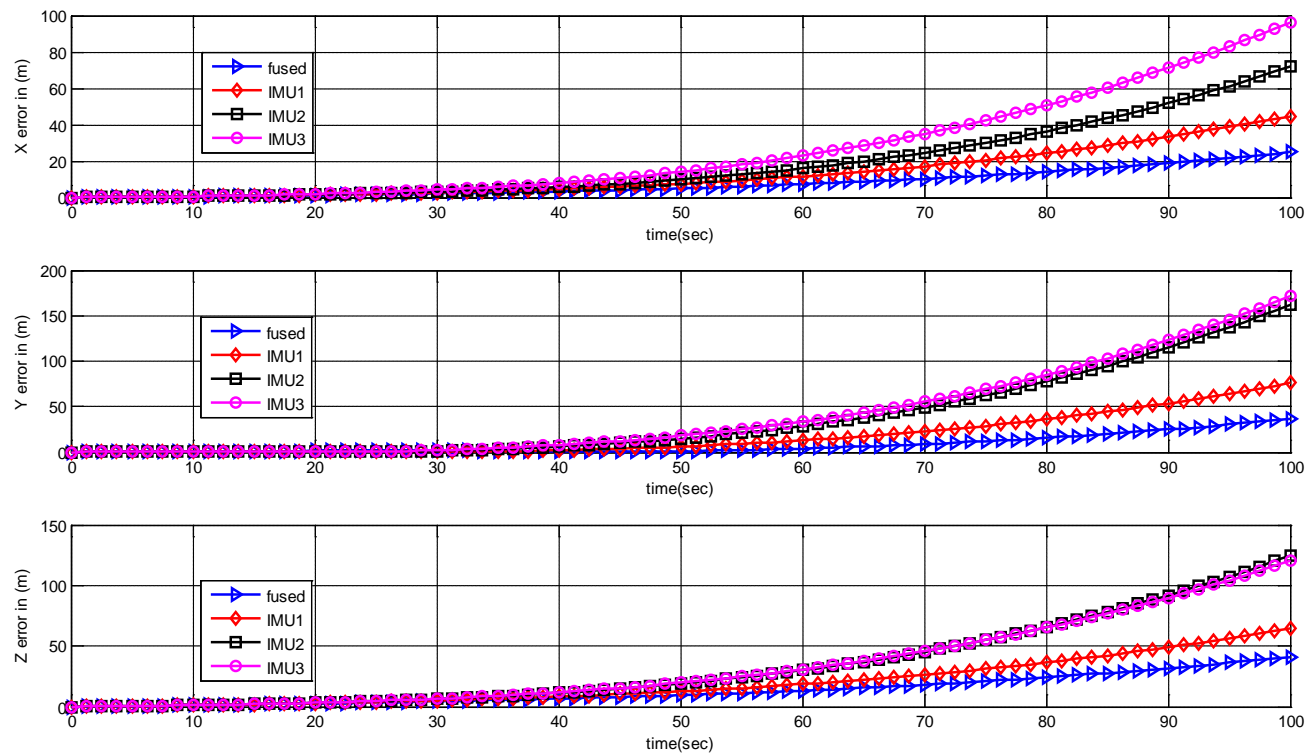

Figure 9. Position error in XYZ 


\section{The Velocity Errors}

\section{The north velocity error}

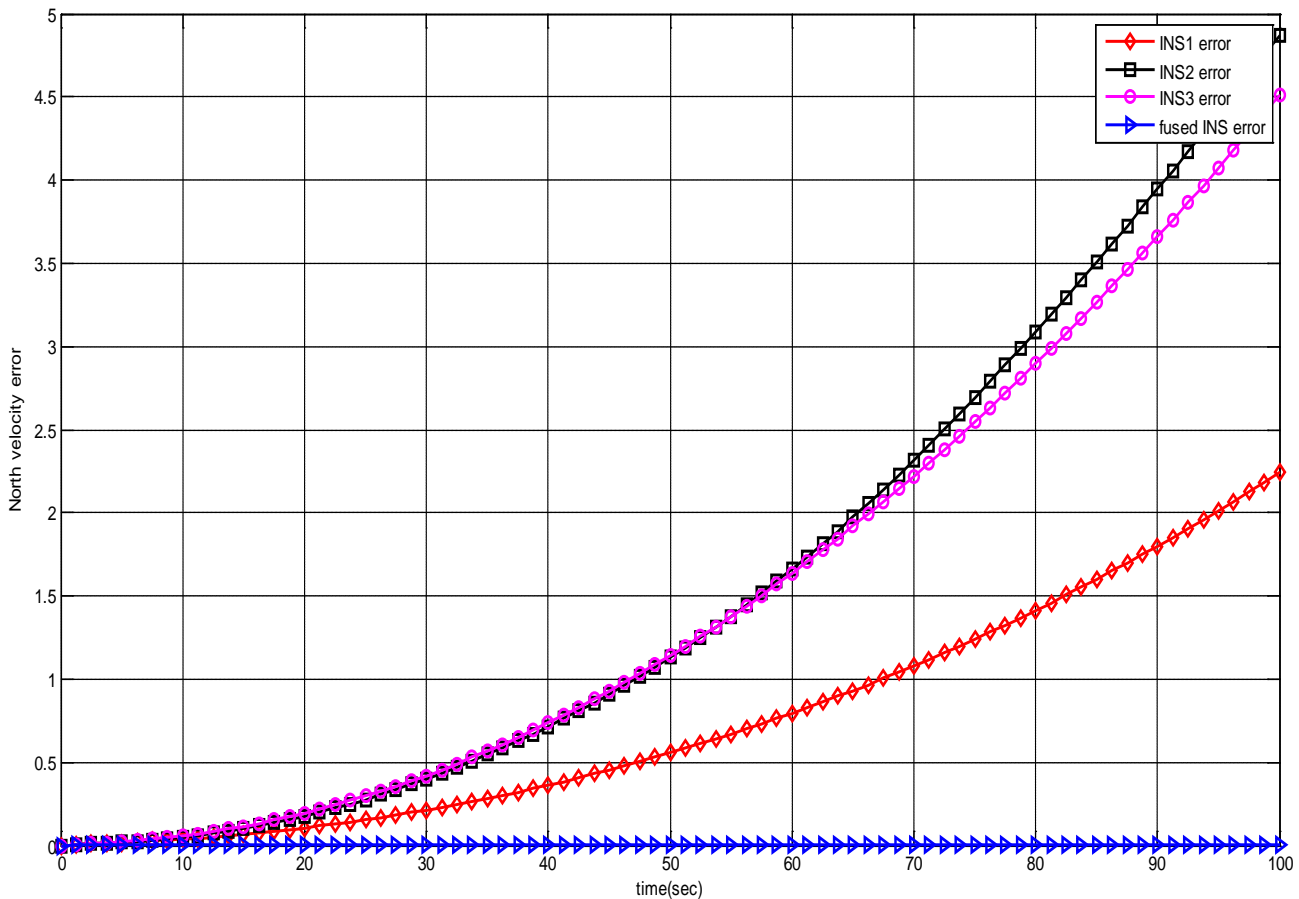

Figure 10. The north velocity error

\section{The east velocity error}

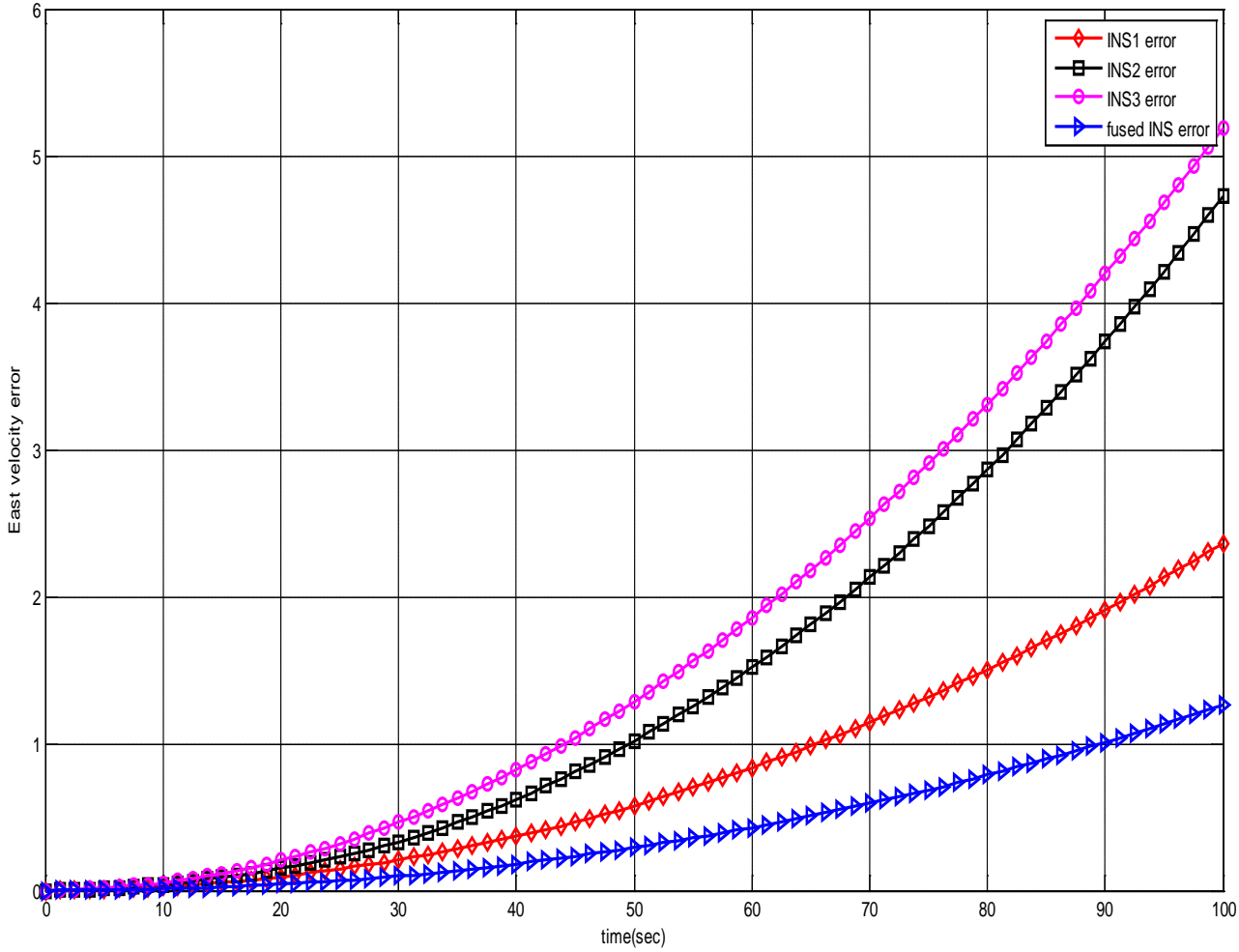

Figure 11. The east velocity error 


\section{The down velocity error}

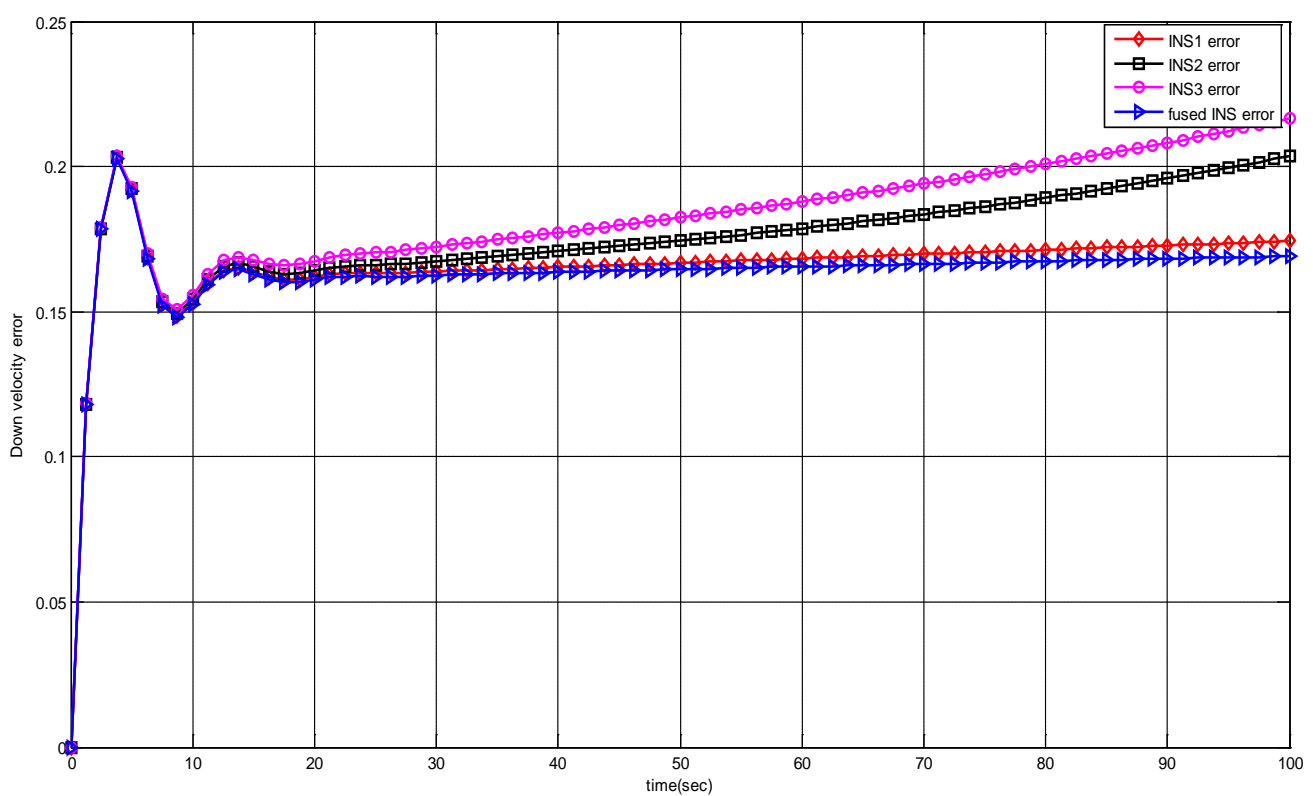

Figure 12. The down velocity error

As shown in Figures (10, 11, and 12) the velocity errors (North, East, and Down) are increasing with time but the error due to the use of the $1^{\text {st }}$ IMU, $2^{\text {nd }}$ IMU, and $3^{\text {rd }}$ IMU is increasing in a higher rate than the error of using the fusion of them. This indicates that the use of multi-sensor data fusion in navigation is better than the use of a single sensor.

\section{The Attitude Errors}
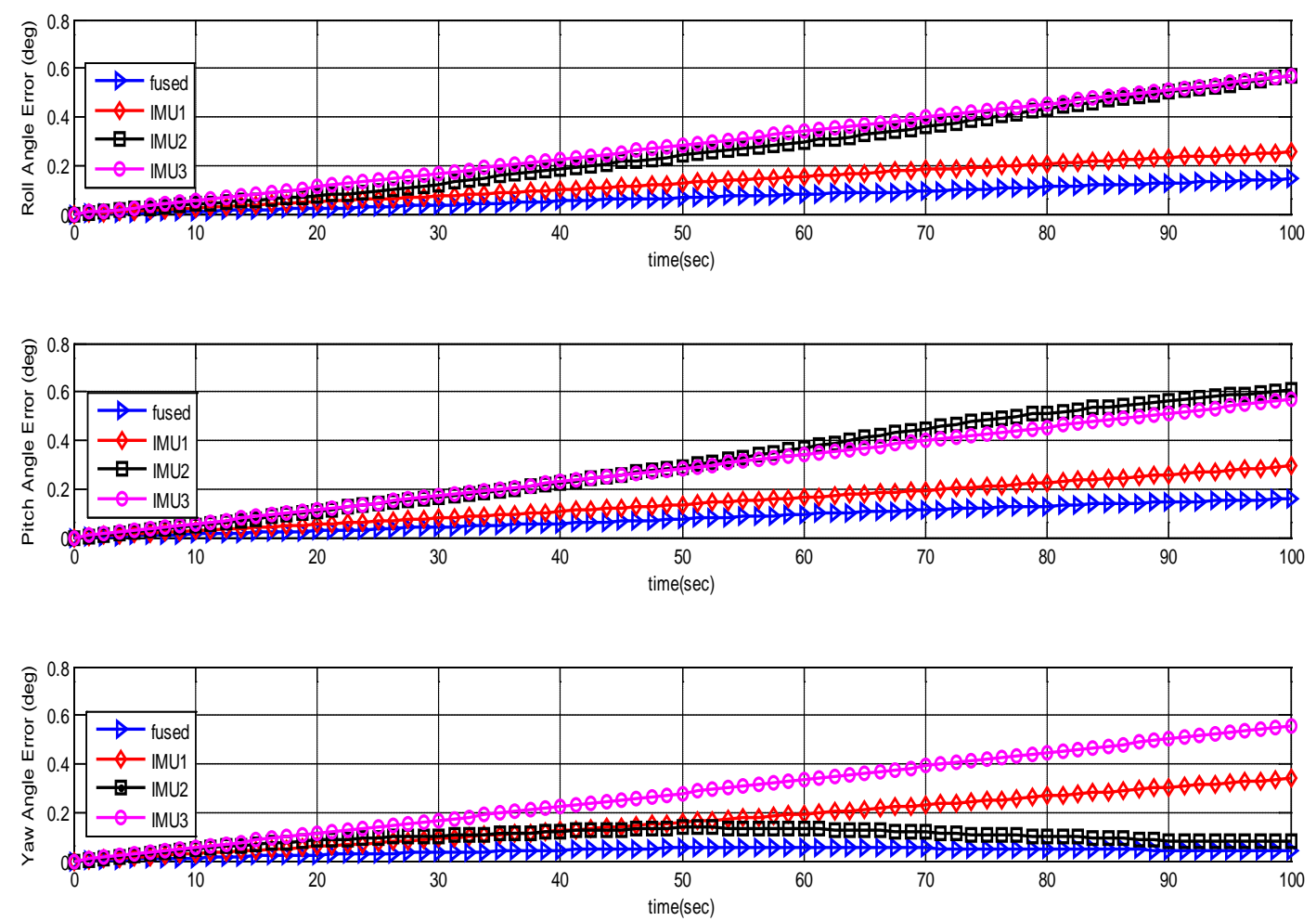

Figure 13. (Roll, pitch, and yaw) Euler angles errors 
As shown in Figure 13 The Euler angles error in (roll, pitch, and yaw) angles are minimum when using multi-sensor data fusion system compared with the errors when using any one of the three IMUs alone.

\section{The Flight Path with and without Fusion}

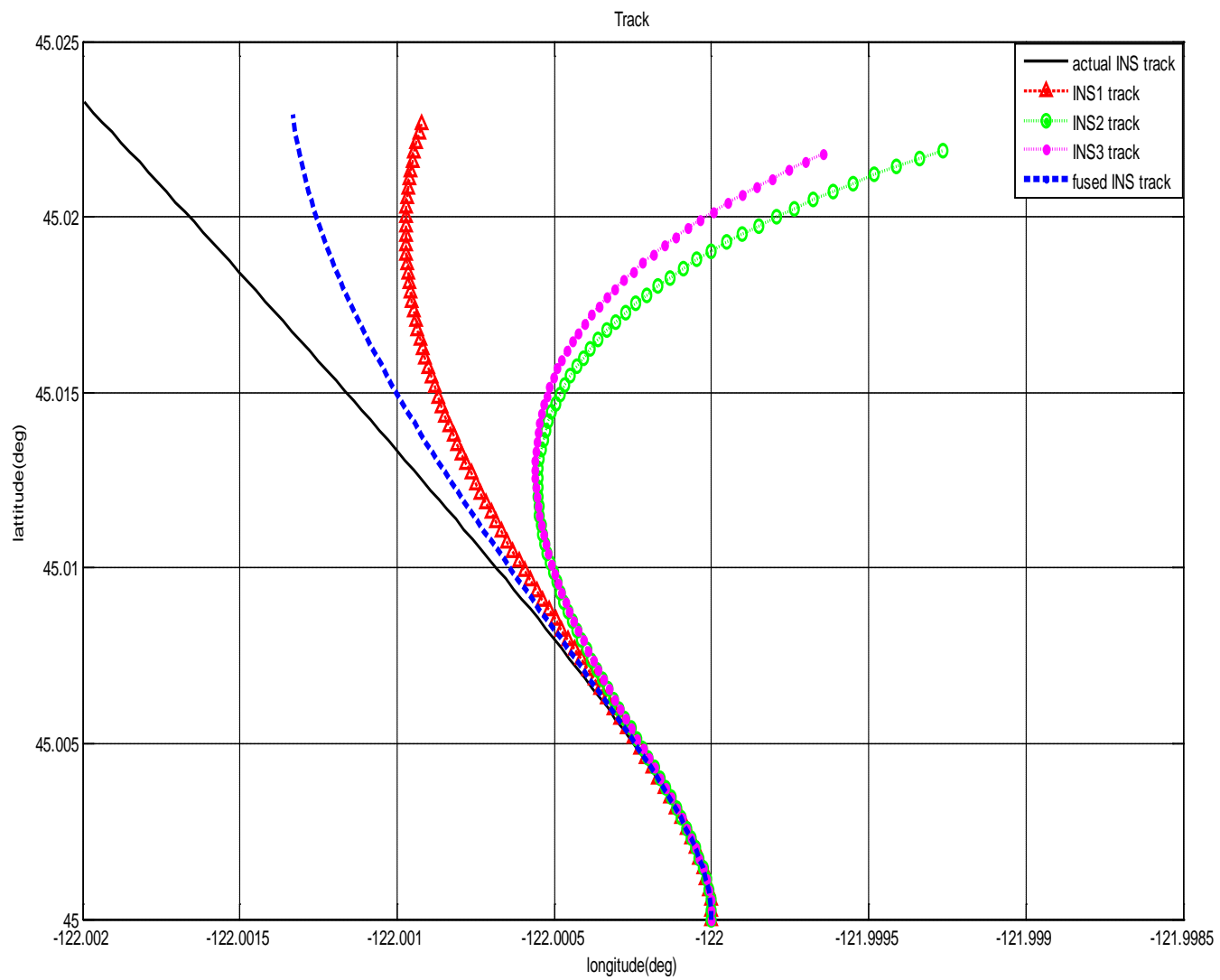

Figure 14. Flight path

In Figure 14 the true flight path is represented by a solid line, the path using the fusion by a dashed line, the path using the $1^{\text {st }}$ IMU is marked with triangle and labeled INS1, the path using the $2^{\text {nd }}$ IMU is marked with circles and labeled INS2, and the path using the $3^{\text {rd }}$ IMU is marked with dots and labeled INS3. The path using the fused data is the closest one to the true path with the minimum error, but the paths of the use of each IMUs alone are far away from the true path. This indicates that the use of multi-sensor data fusion in the aircraft navigation is better than the use of single inertial sensor to produce the navigation information.

\section{Conclusion}

In this paper complete navigation process is achieved, an error model is simulated to be added to the rates and accelerations of the Aerosonde UAV model, and a multi-sensor data fusion approach based on the FCM data clustering is introduced .It does not need any prior data about the sensors signals statistics or the system behavior, and no learning processes are required .This approach indicates strength in navigation of the UAV by reducing the uncertainty of the measured data, hence reducing error of the navigation information compared to the single sensor system. 


\section{References}

[1] Marin E.Liggins, David L.Hall,James LIinas, Handbook of Multisensor Data Fusion Theory and Practice, Second ed.: CRC Press, 2009.

[2] Jitendra R. Raol, Multi-SensorData Fusion with MATLAB. Boca Raton, London, New York: CRC Press, 2010.

[3] Huamin Jia, "Data Fusion Methodologies for Multisensor Aircraft Navigation Systems," phD, College of Aeronautics, Cranfield, Cranfield, 2004.

[4] MOHINDER S. GREWAL, LAWRENCE R. WEILL, ANGUS P. ANDREWS,, GLOBAL POSITIONING SYSTEMS,INERTIAL NAVIGATION,AND INTEGRATION, $2^{\text {nd }}$ ed.: WILEY, 2007.

[5] Eun-Hwan Shin, "Accuracy Improvement of Low Cost INS/GPS for Land Applications," Msc., Calgary, Alberta, 2001.

[6] John Schleppe, "Development of a Real-time Attitude System Using a Quaternion Parameterization and Non-Dedicated GPS Receivers," Msc., Calgary, Alberta, 1996.

[7] Lee, Hyung Keun,Jang Gyu,Yong,Kyu Roh,Park,Chan Gook,, "Modeling quaternion errors in SDINS: computer frame approach," IEEE Transactions on Aerospace and Electronic Systems,, vol. 34, No. 1, pp. 289-300, January 1998.

[8] Savage P. G "Strapdown inertial navigation integration algorithm design part 2: Velocity and position," Guidance Control and Dynamics, vol. 21NO. 2, pp. 208-221, March-April, 1998.

[9] Umanned Dynamics. AeroSim aeronautical simulation blockset Version 1.2. Available: http://www.u-dynamics.com.

[10] Jasbir Singh Vig, "Improved Navigation of a Vehicle Using INS/GPS with Line Of Sight Measurements " master Msc. Thesis, The State University of New York at Buffalo, 2005.

[11] T. Runkler, M. Sturm, and H. Hellendoorn "Model based sensor fusion with fuzzy clustering," IEEE International Conference on Fuzzy Systems, IEEE World Congress on Computational Intelligence, vol. 2, pp. 1377-1382, 1998.

[12] J.C Bezdek, R. Ehrlich , W. Full, "FCM: the fuzzy c-means clustering algorithm," Computers \& Geosciences, vol. 10, pp. 191-198, 1984.

[13] J.C Bezdek, R. Hathaway, M. Sabin ,W. Tucker, "Convergence theory for fuzzy cmeans: counterexamples and repairs," IEEE Transactions on Systems, Man and Cybernetics, vol. 17, pp. 873-877, 1987.

[14] J.C Bezdek, Pattern Recognition with Fuzzy Objective Function Algorithms: Plenum Press, 1981.

[15] J.C Bezdek , J.C Dunn, "Optimal fuzzy partitions: A heuristic for estimating the parameters in a mixture of normal dustrubutions," IEEE Transactions on Computers, pp. 835-838, 1975.

[16] Mohammad Abdel Kareem Jaradat , Reza Langaria,, "A hybrid intelligent system for fault detection and sensor fusion," ELSEVIER, pp. 415-422, 9/ 2009.

[17] R. Duda, P. Hart, D. Stork, Pattern Classification. New York: Wiley, 2001.

[18] C. Bishop, Neural Networks for Pattern Recognition. NewYork: Oxford University Press, 1995. 